\title{
Ventilação não-invasiva com pressão positiva em pacientes com insuficiência respiratória aguda: fatores associados à falha ou ao sucesso*
}

\begin{abstract}
Marcelo Alcantara Holanda ${ }^{1}$, Carlos Henrique Oliveira ${ }^{2}$, Emília Matos Rocha ${ }^{2}$, Rita Moara Bandeira ${ }^{2}$,

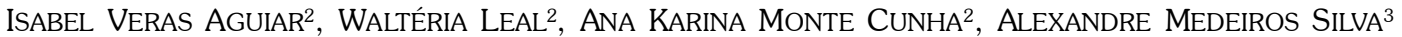

Objetivo: Determinar a eficiência da ventilação não-invasiva com pressão positiva (VNIPP) na insuficiência respiratória aguda e identificar fatores associados ao sucesso ou falha. Pacientes $e$ métodos: Estudo aberto e prospectivo analisando 60 episódios de uso de VNIPP em 53 pacientes em insuficiência respiratória. Resultados: Em 37 episódios (62\%) obteve-se sucesso sem intubação (grupo Sucesso, GS), enquanto em 23 ocasiões (38\%) os pacientes foram intubados (grupo Falha, GF). Os pacientes do GF apresentaram escore de Apache II mais elevado do que os do GS (30,4 \pm 9 versus $22,2 \pm 8, \mathrm{p}=0,001)$. Após $2 \mathrm{~h}$ de VNIPP houve redução da freqüência respiratória, sendo menos intensa no GF (de $33 \pm 9$ para $30 \pm 8$ irpm, $\mathrm{p}=0,094$ ) do que no GS (de $39 \pm 11$ para $28 \pm 9$ irpm, $\mathrm{p}<0,001$ ). Houve aumento da $\mathrm{PaO}_{2}$ (de $62 \pm 22$ para $101 \pm 65 \mathrm{mmHg}, \mathrm{p}<0,001$ ), sem diferenças entre os grupos. Nos pacientes com hipercapnia houve redução da $\mathrm{PaCO}_{2}$ no GS (de $76 \pm 20$ para $68 \pm 21 \mathrm{mmHg}, \mathrm{p}=0,032)$ e não no GF (de $89 \pm 23$ para $93 \pm 40 \mathrm{mmHg}, \mathrm{p}=0,54)$. O pH se elevou de 7,25 $\pm 0,10$ e 7,34 $\pm 0,11, p=0,007$ no GS, mas não no GF $(7,24 \pm 0,07$ e 7,21 $\pm 0,12$, $\mathrm{p}=0,48)$. A VNIPP foi utilizada por mais tempo no GS $(3,4 \pm 2,5$ versus $2,3 \pm 2$ dias, $p=0,003) e$ com níveis mais altos de pressão respiratória positiva em via aérea (IPAP) (13,2 \pm 3 versus

$\left.11 \pm 4 \mathrm{cmH}_{2} \mathrm{O}, \mathrm{p}=0,02\right)$. Dez pacientes (17\%) foram a óbito, todos no GF. A complicação mais freqüente foi lesão de pele no ponto de contato da máscara com o nariz $(5,8 \%)$. A VNIPP foi eficiente no tratamento da insuficiência respiratória aguda em cerca de $2 / 3$ das vezes. Pacientes mais graves, menor eficiência em reduzir a freqüência respiratória, em reverter a acidose respiratória e o uso de níveis relativamente mais baixos de pressão respiratória positiva em via aérea (IPAP) associaram-se à falha. A alta mortalidade $(10,43 \%)$ nos casos de falha justifica esforços para otimizar sua utilização e ao mesmo tempo para reconhecer precocemente suas falhas, evitando-se protelar a intubação traqueal. (J Pneumol 2001;27(6):301-309)

\section{Non-invasive positive pressure ventilation in patients with acute respiratory failure: factors associated with failure or success}

Objective: To evaluate the effects of non-invasive positive pressure ventilation (NIPPV) on acute respiratory failure and to identify the factors associated with its failure or success. Patients and methods: This open and prospective study analyzed 60 episodes of NIPPV use in 53 patients with respiratory failure. Results: In 37 occasions $(62 \%)$, the patients were successfully ventilated with NIPPV (Success Group, SG) whereas in 23 episodes (38\%) intubation was necessary (Failure Group, FG). FG patients showed a higher Apache II score (30.4 \pm 9 versus $22.2 \pm 8, p=0.001)$. The use of NIPPV reduced respiratory rate after 2 hs. This reduction was less evident in FG (from $33 \pm 9$ to 30 $\pm 8 \mathrm{bpm}, \mathrm{p}=0.094$ ) than in SG (from $39 \pm 11$ to $28 \pm 9 \mathrm{bpm}, \mathrm{p}<0.001$ ). $\mathrm{PaO}_{2}$ increased from $62 \pm 22$ to $101 \pm 65 \mathrm{mmHg}$, $(\mathrm{p}<0.001)$ with no differences between groups. Among patients

* Trabalho realizado na Unidade de Terapia Intensiva Respiratória do Hospital de Messejana, Fortaleza, CE.

1. Coordenador da Residência de Pneumologia; Médico diarista da UTI respiratória.

2. Fisioterapeuta Respiratório da UTI respiratória.

3. Médico Pneumologista da UTI respiratória.
Endereço para correspondência - Dr. Marcelo Alcantara Holanda, Rua Coronel Jucá, 700, casa 30 - 60170-320 - Fortaleza, CE. Tels. (85) 267-7220 ou 454-2323; Fax (85) 254-7910; E-mail: holand@secrel. com.br

Recebido para publicação em 7/12/00. Aprovado, após revisão, em 19/4/01. 
with hypercapnia, $\mathrm{PaCO}_{2}$ level decreased in SG (from $76 \pm 20$ to $68 \pm 21 \mathrm{mmHg}, \mathrm{p}=0.032$ ) but not in FG (from $89 \pm 23$ to $93 \pm 40 \mathrm{mmHg}, \mathrm{p}=0.54$ ). Arterial pH increased in SG (from $7.25 \pm$ 0.10 to $7.34 \pm 0.11, p=0.007$ ) and not in FG (from $7.24 \pm 0.07$ to $7.21 \pm 0.12, p=0.48$ ). NIPPV was used for longer in SG $(3.4 \pm 2.5$ versus $2.3 \pm 2$ days, $p=0.003)$. Higher IPAP levels were used in SG $\left(13.2 \pm 3\right.$ versus $\left.11 \pm 4 \mathrm{cmH}_{2} \mathrm{O}, \mathrm{p}=0.02\right)$. The 10 patients $(17 \%)$ who died, all belonged to FG. Skin lesions at the site of mask contact was the most common complication of NIPPV $(5,8 \%)$. NIPPV was efficient in treating acute respiratory failure in $2 / 3$ of patients. The factors associated with failure were: greater severity of the illness, smaller reduction in the respiratory rate, no improvement of respiratory acidosis in $2 \mathrm{~h}$ and use of lower IPAP levels. The high mortality rate observed in FG $(10,43 \%)$ justifies efforts to optimize NIPPV implementation and to recognize its failure, as early as possible, in order to avoid delays in endotracheal intubation.

Descritores - Ventilação não-invasiva com pressão positiva. Insuficiência respiratória. Apache II. Hipercapnia.

Key words - Non-invasive positive pressure ventilation. Respiratory failure. Apache II. Hypercapnia.

\section{INTRODUÇÃO}

A ventilação não-invasiva com pressão positiva (VNIPP) consiste em método de assistência ventilatória em que uma pressão positiva é aplicada à via aérea do paciente através de máscaras ou outras interfaces sem a utilização da intubação traqueal ${ }^{(1)}$. A vNIPP tem uma série de vantagens em relação à ventilação invasiva: é de fácil aplicação e remoção, preserva as vias aéreas superiores, garante maior conforto ao paciente, evita o trabalho resistivo do tubo traqueal e as complicações da própria intubação, como traumatismos de vias aéreas superiores ou pneumonia nosocomial(1-3). Embora já utilizada desde o final da década de 30 por Barach et al. ${ }^{(4)}$, a VNIPP somente veio mostrar-se como alternativa eficaz e vantajosa em relação à intubação traqueal no início dos anos 90. Desde então, um grande número de artigos tem sido publicado, demonstrando não só sua eficiência em prevenir a intubação traqueal, mas também seus efeitos em diminuir as complicações decorrentes da intubação e em reduzir a mortalidade de pacientes em insuficiência respiratória aguda, como os portadores de DPOC ${ }^{(3,5-7)}$. Em recente metanálise, Keenan et al. ${ }^{(8)}$ demonstraram o impacto da VNIPP na redução da necessidade de intubação traqueal e da mortalidade em pacientes com insuficiência respiratória. Os resultados da literatura e o aprimoramento dos materiais utilizados (máscaras, outras interfaces e ventiladores geradores de fluxo) têm expandido o uso da VNIPP em nosso meio ${ }^{(9,10)}$. Contudo, a VNIPP também apresenta desvantagens em relação à ventilação invasiva: correção mais lenta dos distúrbios de troca gasosa, necessidade de maior número de profissionais à beira do leito para sua implementação, problemas com as interfaces (vazamentos, ina-
Siglas e abreviaturas utilizadas neste trabalho

Apache II - Acute Physiology and Chronic Health Evaluation II CPAP - Pressão positiva contínua em via aérea

EPAP - Pressão expiratória positiva em via aérea

$\mathrm{f}$ - Freqüência respiratória

$\mathrm{FIO}_{2}$ - Fração inspirada de oxigênio

IPAP - Pressão inspiratória positiva em via aérea

PS - Pressão de suporte

$\mathrm{SpO}_{2}$ - Saturação da hemoglobina com oxigênio determinada por oxímetro de pulso

VNIPP - Ventilação não-invasiva com pressão positiva

daptação) e dificuldades no acesso às vias aéreas inferiores, sobretudo em pacientes com hipersecreção brônquica. Além disso, o candidato ideal para a VNIPP necessita estar consciente, alerta, cooperativo (excetuando pacientes com narcose por hipercapnia), hemodinamicamente estável e sem dificuldades para adaptação à máscara $e$ ao modo ventilatório empregado(3). Provavelmente por essas limitações da VNIPP, são descritos insucessos em taxas que variam de 5 a $40 \%^{(2,3,8)}$. O reconhecimento dos pacientes com maior risco de insucesso à VNIPP é importante, pois pode evitar a insistência nesse tipo de suporte ventilatório e o retardo na intubação traqueal, levando a manejo inadequado dos pacientes em insuficiência respiratória.

O presente trabalho teve por objetivos: 1) determinar a eficiência da VNIPP em pacientes com insuficiência respiratória aguda de diversas etiologias; 2 ) identificar fatores associados ao sucesso ou ao insucesso da VNIPP; 3) caracterizar os padrões de resposta à VNIPP, seu modo de uso $e$ complicações associadas.

\section{MATERIAL E MÉTODOS}

Este estudo prospectivo e aberto foi realizado em um período de dois anos, de outubro/1998 a setembro/2000, na UTI respiratória do Hospital de Messejana, Fortaleza, CE. A UTI respiratória consta de uma unidade de cinco 
leitos em hospital terciário especializado em doenças do tórax e com características de hospital-escola para residentes de pneumologia, cardiologia e cirurgia cardiovascular, além de especialização em fisioterapia cardiorrespiratória. O trabalho foi aprovado pelo Comitê de Ética em Pesquisa e seguiu as normas das Resoluções 96/196 e 251/97 do Conselho Nacional de Saúde.

Os critérios de inclusão foram: insuficiência respiratória aguda com provável necessidade de assistência ventilatória segundo, pelo menos, dois dos seguintes critérios: a) f > 25irpm; b) uso de musculatura acessória ou respiração paradoxal; c) $\mathrm{PaO}_{2}<60 \mathrm{mmHg}$ ou $\mathrm{SaO}_{2}$ ou $\mathrm{SpO}_{2}<$ 90\% em ar ambiente ou mesmo em oxigenioterapia; d) $\mathrm{PaCO}_{2}>46 \mathrm{mmHg}$ com $\mathrm{pH} \leq 7,33$; e) insuficiência respiratória pós-extubação. Foram os seguintes os critérios de exclusão: necessidade de intubação traqueal imediata por parada respiratória iminente; instabilidade hemodinâmica ou arritmias cardíacas; escore de coma de Glasgow < 10 ou paciente não cooperativo (excetuando os casos de narcose por hipercapnia); excesso de secreção traqueobrônquica; hemorragia digestiva alta e traumas de face. A VNIPP foi implementada pela equipe de fisioterapia, enfermagem e médica, sendo utilizados quatro diferentes ventiladores, conforme a disponibilidade no momento da inclusão do paciente: Bilevel ${ }^{\circledR}$ (DeVilbiss, Pennsylvania, EUA), Bird 8400STi ${ }^{\circledR}$, Bird $6400^{\circledR}$ (Bird CO, Palm Springs, CA, EUA) e Inter $5^{\circledR}$ (Intermed, São Paulo, Brasil). A escolha do ventilador obedeceu aos seguintes critérios: o ventilador Bileve ${ }^{\circledR}$ era usado como primeira opção $e$, caso não estivesse disponível no momento por estar sendo utilizado em outro paciente, optava-se pelos ventiladores Bird $8400 \mathrm{STi}^{\circledR}$, Bird $6400^{\circledR} e$ Inter $5^{\circledR}$, nesta ordem de preferência. Além disso, nos casos de hipoxemia grave em que houve necessidade de $\mathrm{FIO}_{2}>50 \%$ optou-se pelos ventiladores microprocessados convencionais, por garantirem $\mathrm{FIO}_{2}$ até $100 \%$, se necessário. Após explicação do procedimento ao paciente, este era submetido a um atendimento de fisioterapia respiratória para drenagem de secreções, se necessário, e posteriormente mantido em posição de Fowler (inclinação de $45^{\circ}$ ). A seguir, a interface era escolhida, preferindo-se inicialmente a máscara nasal (Downs CPAP Mask ${ }^{\circledR}$, Vital Signs, Hong Kong) e, se ineficiente ou naqueles pacientes com respiração predominantemente bucal, a máscara facial 7355 Series $^{\circledR}$ (Somerset, Pensilvânia, EUA). Independente do tipo escolhido, a máscara era adaptada à face do paciente manualmente e de modo gentil, até que o mesmo estivesse em sincronia com o ventilador. Somente então era fixada à cabeça do paciente. No ventilador Bilevel ${ }^{\circledR}$ a VNIPP era iniciada com IPAP (pressão inspiratória positiva em via aérea) de 5 a $7 \mathrm{cmH}_{2} \mathrm{O}$ e EPAP (pressão expiratória positiva em via aérea) de $3 \mathrm{cmH}_{2} \mathrm{O}$ com $\mathrm{O}_{2}$ ofertado por fluxo externo suficiente para man- ter a $\mathrm{SpO}_{2} \geq 92 \%$. Quando utilizados os ventiladores Bird $8400 S T i^{\circledR}$, Bird $6400^{\circledR}$ e Inter $5^{\circledR}$, o modo CPAP + PS (pressão positiva contínua em via aérea + pressão de suporte) era ajustado com níveis iniciais de CPAP de $3 \mathrm{cmH}_{2} \mathrm{O}$ $e$ PS de 4 a $5 \mathrm{cmH}_{2} \mathrm{O}$, sensibilidade à pressão $\left(1 \mathrm{a} 2 \mathrm{cmH}_{2} \mathrm{O}\right)$ ou a fluxo $(2 \mathrm{~L} / \mathrm{min})$ e $\mathrm{FIO}_{2}$ ajustada para manter a $\mathrm{SpO}_{2}$ $\geq 92 \%$. Os pacientes foram analisados globalmente e em dois subgrupos: grupo Sucesso (GS), em que a VNIPP obteve sucesso em evitar a intubação, e grupo Falha (GF), que incluiu todos os pacientes que foram intubados após a tentativa inicial com VNIPP. Foram analisados em separado os pacientes com insuficiência respiratória com hipercapnia, bem como os com acidose respiratória. Os seguintes parâmetros foram registrados em ficha apropriada para a VNIPP: idade, sexo, diagnóstico, escore Apache II, freqüência respiratória, uso de musculatura acessória, movimento paradoxal, interface utilizada (máscara facial ou nasal), níveis de IPAP, EPAP e fluxo externo de $\mathrm{O}_{2}$ se utilizado o ventilador Bileve ${ }^{\circledR}$, e ajustes de PS, PEEP $e$ $\mathrm{FIO}_{2}$, quando utilizados os ventiladores Bird 8400STi ${ }^{\circledR}$, Bird $6400^{\circledR}$ e Inter $5^{\circledR}$. Os pacientes foram seguidos durante todo o período em que utilizaram a VNIPP, sendo registradas: duração da VNIPP, causas de falha, indicações de intubação, complicações, incluindo óbitos e mudanças nos ajustes do ventilador. Durante a VNIPP a seguinte estratégia foi adotada: coleta seriada de gasometria arterial, aumento da IPAP ( $2 \mathrm{a} 3 \mathrm{cmH}_{2} \mathrm{O}$ por vez), se verificada persistência da acidose respiratória ou desconforto respiratório, e aumento da $\mathrm{FIO}_{2}$ e/ou da EPAP, se hipoxemia persistente com $\mathrm{SaO}_{2}$ ou $\mathrm{SpO}_{2}<92 \%$ e/ou $\mathrm{PaO}_{2}<60$ $\mathrm{mmHg}$. A decisão quanto à intubação dos pacientes coube ao médico responsável pelos mesmos no exato instante em que esse procedimento se fez necessário, obedecendo a critérios clínicos usuais e correntemente praticados em nossa instituição, a saber: agravamento da doença de base, piora do nível de consciência com escore de coma de Glasgow < 10, instabilidade hemodinâmica ou arritmias cardíacas, agitação que precisasse de sedação, incapacidade do paciente em eliminar secreções, piora da acidose respiratória apesar de repetidos ajustes na VNIPP, hipoxemia refratária $\left(\mathrm{SpO}_{2}<92 \%\right.$ ou $\mathrm{PaO}_{2}<60$ $\mathrm{mmHg}$ ) e risco de parada cardiorrespiratória iminente. A retirada da VNIPP deu-se de forma gradual com interposição de períodos de ventilação espontânea progressivamente mais longos até sua retirada total, desde que controlada a causa básica da insuficiência respiratória. O paciente foi considerado "desmamado" da VNIPP se permanecesse por mais de $48 \mathrm{~h}$ em ventilação espontânea.

Análise estatística - Os grupos Sucesso (GS) e Falha (GF) foram comparados entre si pelo método do qui-quadrado ou teste exato de Fisher para variáveis categóricas e pelo teste t de Student para variáveis contínuas. As variáveis contínuas dentro de cada grupo foram compara- 
das pelo teste t pareado e bicaudal. Foi determinado valor de 0,05 como nível de significância para rejeição da hipótese de nulidade.

\section{REsUlTADOs}

No período estudado, 190 pacientes foram admitidos em respiração espontânea na UTI respiratória. A VNIPP foi usada como técnica de assistência ventilatória em 45 ocasiões $(24 \%)$, em que foram preenchidos os critérios de inclusão. Em outros 15 episódios a VNIPP foi empregada como assistência ventilatória pós-extubação ( $20 \%$ de um total de 74 pacientes extubados no período). Foi analisado, portanto, um total de 60 episódios consecutivos de insuficiência respiratória tratados com a VNIPP, em 53 pacientes. Sete indivíduos fizeram uso da VNIPP em duas ocasiões distintas. A VNIPP obteve sucesso em evitar a intubação em 37 episódios (62\%), constituindo o grupo Sucesso (GS). Em 23 ocasiões houve necessidade de intubação traqueal configurando falha da VNIPP (grupo Falha, GF). A Tabela 1 resume as principais características clínicas dos pacientes quando da inclusão no estudo. O diagnóstico mais comum foi de DPOC $(19,32 \%)$ seguido de insuficiência respiratória pós-extubação $(15,25 \%)$ e pneu- monia $(6,10 \%)$. Não houve diferenças significativas com relação ao sexo, idade, diagnóstico, presença de hipercapnia e escore de coma de Glasgow. Não houve diferenças quanto à freqüência cardíaca basal ou dados de gasometria arterial antes da VNIPP. Somente o escore de Apache II foi significativamente mais elevado no grupo Falha (30 \pm 9 versus $22 \pm 8, p=0,001$ ).

As variações da freqüência respiratória e dos parâmetros gasométricos são apresentados nas Figuras de 1 a 4 . Os pacientes que responderam (GS) apresentaram tendência a maior freqüência respiratória basal antes da VNIPP do que o grupo Falha $(39 \pm 11$ versus $33 \pm$ 9irpm $p=$ $0,068)$. A VNIPP proporcionou redução significativa na freqüência respiratória após duas horas quando analisados todos os pacientes (de $37 \pm 10$ para $29 \pm$ 9irpm, $\mathrm{p}<$ 0,001 ). No grupo Sucesso essa redução foi mais acentuada (de $39 \pm 11$ para $28 \pm 9, \mathrm{p}<0,001$ ) do que no grupo Falha (de $33 \pm 9$ para $30 \pm 8$ irpm, $p=0,094$ ) (Figura 1). A melhora da $\mathrm{PaO}_{2}$ nos episódios em que foi colhida gasometria arterial antes e após $2 \mathrm{~h}$ de VNIPP ( $\mathrm{n}=$ $49,82 \%$ ), ocorreu de modo significativo quando analisados todos os pacientes (de $62 \pm 22$ para $101 \pm 65 \mathrm{mmHg}$, $p<0,001)$ bem como nos GS e GF analisados separadamente (Figura 2). Com relação aos pacientes com hiper-

TABELA 1

Características dos pacientes por ocasião de sua inclusão no estudo segundo os grupos Sucesso (GS) e Falha (GF)

\begin{tabular}{|c|c|c|c|c|c|c|c|}
\hline \multirow[b]{2}{*}{ Sexo: } & \multicolumn{2}{|c|}{ GS, $\mathbf{n}=37$} & \multicolumn{2}{|c|}{$G F, n=23$} & \multicolumn{2}{|c|}{ Todos, $n=60$} & \multirow[t]{2}{*}{ Valor de $\mathrm{p}^{*}$} \\
\hline & & & & & & & \\
\hline Masculino & \multirow{2}{*}{\multicolumn{2}{|c|}{$\begin{array}{l}21(57 \%) \\
16(43 \%)\end{array}$}} & \multirow{2}{*}{\multicolumn{2}{|c|}{$\begin{array}{l}12(52 \%) \\
11(48 \%)\end{array}$}} & \multirow{2}{*}{\multicolumn{2}{|c|}{$\begin{array}{l}33(55 \%) \\
27(45 \%)\end{array}$}} & 0,89 \\
\hline Feminino & & & & & & & \\
\hline Idade & 52 & 19 & 60 & 21 & 55 & 20 & 0,15 \\
\hline Apache II & 22,2 & 8,3 & 30,4 & 8,9 & 25,3 & 9,4 & 0,001 \\
\hline Escore de Glasgow & 14,5 & 1,5 & 13,6 & 2,5 & 14,2 & 1,9 & 0,12 \\
\hline FC basal (bpm) & 118 & 22 & 124 & 23 & 120 & 22 & 0,34 \\
\hline Presença de hipercapnia n (\%) & \multicolumn{2}{|c|}{$23(62 \%)$} & \multicolumn{2}{|c|}{$15(65 \%)$} & \multicolumn{2}{|c|}{$38(63 \%)$} & 0,81 \\
\hline \multicolumn{8}{|l|}{ Diagnósticos: } \\
\hline Exacerbação de DPOC & \multicolumn{2}{|c|}{$10(27 \%)$} & \multicolumn{2}{|c|}{$9(39 \%)$} & \multicolumn{2}{|c|}{$19(32 \%)$} & 0,33 \\
\hline I resp pós-extubação & \multicolumn{2}{|c|}{$9(24 \%)$} & \multicolumn{2}{|c|}{$6(26 \%)$} & \multicolumn{2}{|c|}{$15(25 \%)$} & 0,92 \\
\hline Pneumonia & \multicolumn{2}{|c|}{$4(11 \%)$} & \multicolumn{2}{|c|}{$2(9 \%)$} & \multicolumn{2}{|c|}{$6(10 \%)$} & NS \\
\hline Bronquiectasia & \multicolumn{2}{|c|}{$2(6 \%)$} & \multicolumn{2}{|c|}{0} & \multicolumn{2}{|c|}{$2(3 \%)$} & NS \\
\hline Cifoescoliose & \multicolumn{2}{|c|}{$1 \quad(3 \%)$} & \multicolumn{2}{|c|}{$2(9 \%)$} & \multicolumn{2}{|c|}{$3(5 \%)$} & NS \\
\hline Asma & \multicolumn{2}{|c|}{$2(5 \%)$} & \multicolumn{2}{|c|}{0} & \multicolumn{2}{|c|}{$2(3 \%)$} & NS \\
\hline ICC + EAP & \multicolumn{2}{|c|}{$1 \quad(3 \%)$} & \multicolumn{2}{|c|}{$2 \quad(9 \%)$} & \multicolumn{2}{|c|}{$3 \quad(5 \%)$} & NS \\
\hline Pneumocistose & \multicolumn{2}{|c|}{$2 \quad(5 \%)$} & \multicolumn{2}{|c|}{0} & \multicolumn{2}{|c|}{$2(3 \%)$} & NS \\
\hline Outros $^{\dagger}$ & & $16 \%)$ & & $(9 \%)$ & & |3\%) & NS \\
\hline
\end{tabular}

FC - freqüência cardíaca; ICC - insuficiência cardíaca congestiva; EAP - edema agudo de pulmão; ${ }^{*} \mathrm{GS}$ versus GF; ${ }^{\dagger}$ Um caso de cada um dos diagnósticos: fibrose pulmonar idiopática, silicose avançada, hipertensão pulmonar, doença neuromuscular, P.O. pneumectomia e seqüela de tuberculose no GS e um caso de empiema + sepse e um de linfoma no GF. 


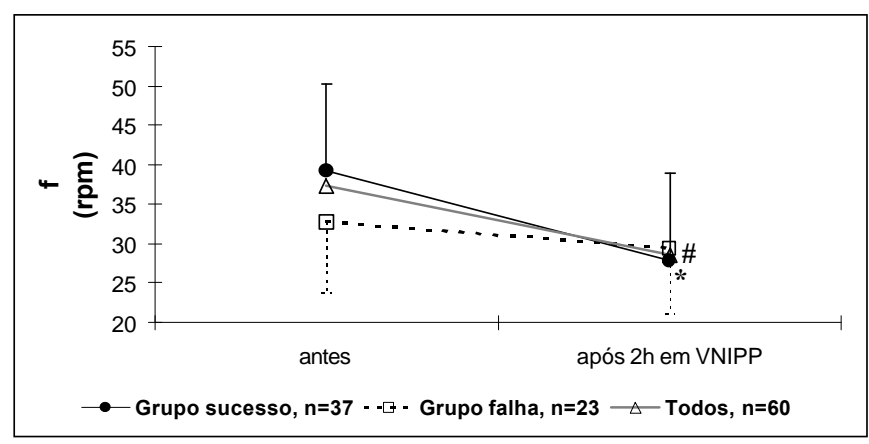

Figura 1 - Variação da freqüência respiratória (média e desvio padrão) nos grupos Sucesso (GS) e Falha (GF) antes e após duas horas em VNIPP. ${ }^{*} p<0,01$ no GS e \# $p<0,01$ em Todos (valores pré versus após-VNIPP).

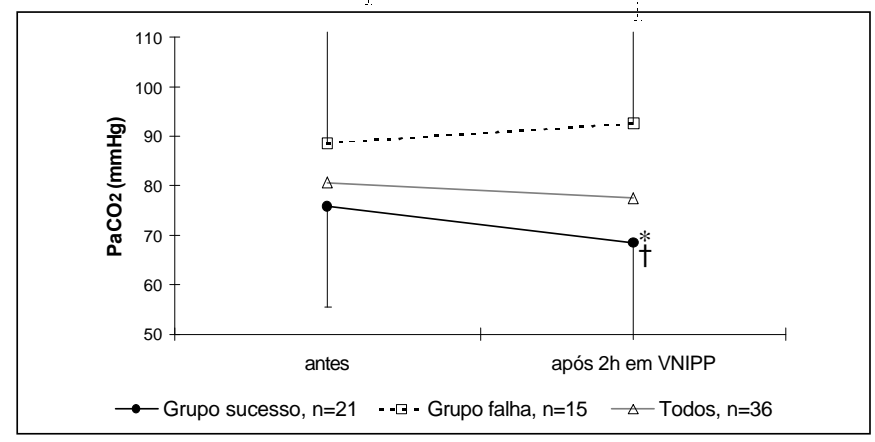

Figura 3 - Variação da $\mathrm{PaCO}_{2}$ (média e desvio padrão) nos grupos Sucesso (GS) e Falha (GF) nos pacientes com hipercapnia antes e após o uso da VNIPP. * $p<0,05$ no GS e $+p<0,05$ GS versus GF.

capnia $(\mathrm{n}=36,60 \%)$ a VNIPP reduziu a $\mathrm{PaCO}_{2}$ somente nos pacientes do grupo Sucesso (76 \pm 20 para $68 \pm 21$ $\mathrm{mmHg}, \mathrm{p}=0,032$ ), mas não no grupo Falha (de $89 \pm 23$ para $93 \pm 40 \mathrm{mmHg}, \mathrm{p}=0,54$ ) (Figura 3 ). O mesmo ocorreu com relação ao $\mathrm{pH}$ nos pacientes com acidose respiratória ( $\mathrm{n}=19,32 \%$ ). Somente no grupo Sucesso houve incremento significativo do $\mathrm{pH}$ ao final de $2 \mathrm{~h}$ de VNIPP (de 7,25 $\pm 0,10$ para 7,34 $\pm 0,11, \mathrm{p}=0,007$ ), o que não se evidenciou no grupo Falha (de 7,24 \pm 0,07 para 7,21 $\pm 0,12, p=0,48$ ) (Figura 4).

A Tabela 2 mostra os dados relacionados ao modo de implementação da VNIPP e quanto à evolução dos pacientes. Houve preferência por utilizar máscara nasal $(n=39$, $65 \%$ ) associada a um ventilador com gerador de fluxo ( $\mathrm{n}$ $=42,70 \%$ ). O nível máximo de IPAP utilizado no grupo Sucesso foi superior ao do grupo Falha $(13,2 \pm 3$ versus $11 \pm 4 \mathrm{cmH}_{2} \mathrm{O}, \mathrm{p}=0,020$ ). O tempo em número de dias de uso da VNIPP foi superior no grupo Sucesso. No grupo Falha a maioria das intubações ocorreu em tempo inferior a $24 \mathrm{~h}(\mathrm{n}=13,57 \%)$, configurando falhas precoces da VNIPP. Somente seis pacientes (26\%) foram intubados após pelo menos três dias de uso da VNIPP, configurando falhas tardias. A causa da intubação mais freqüente foi

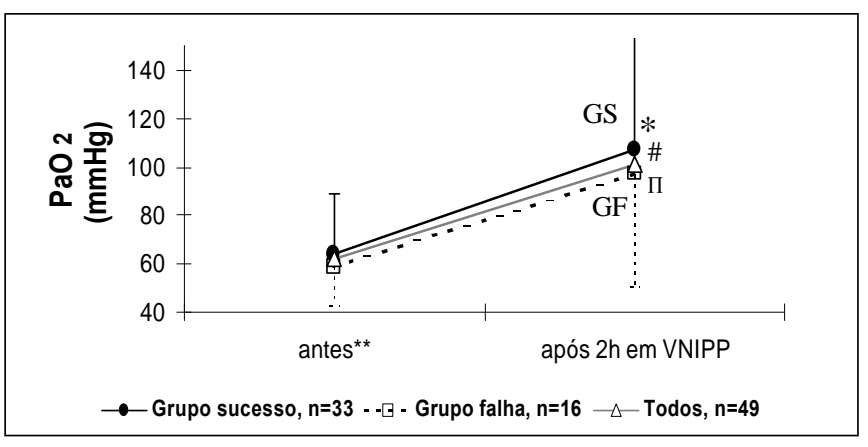

Figura 2 - Variação da $\mathrm{PaO}_{2}$ (média e desvio padrão) nos grupos Sucesso (GS) e Falha (GF) nos pacientes com gasometria arterial antes e após a VNIPP. ${ }^{*} p<0,01$ no GS e \# $p<0,01$ Todos e $\pi p<$ 0,01 no GF. ** em oxigenioterapia (valores pré versus após-VNIPP).

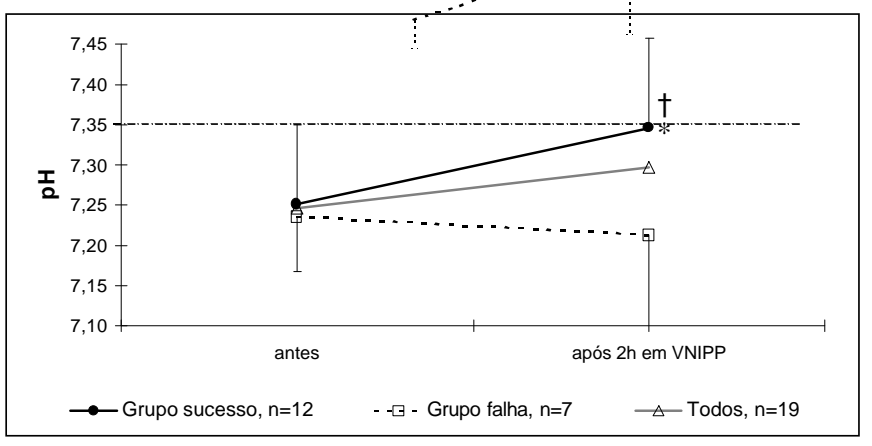

Figura 4 - Variação do $\mathrm{pH}$ (média e desvio padrão) nos grupos Sucesso (GS) e Falha (GF) nos pacientes com acidose respiratória, antes e após o uso da VNIPP. * $p<0,01$ no GS (valores pré versus após-VNIPP) $+p=0,023$ (GS versus GF).

piora da doença de base $(n=14,61 \%)$. As complicações decorrentes diretamente da VNIPP foram relacionadas principalmente à interface utilizada (lesão de pele no ponto de contato com a máscara) em cinco pacientes ( $8 \%$ do total) sendo quatro no GS e um no GF. Os pacientes que foram intubados permaneceram em média $12 \pm 10$ dias em ventilação mecânica invasiva (variando de poucas horas até 38 dias). Nenhum óbito foi registrado no GS, enquanto a mortalidade no GF foi de $43 \%$ (10 pacientes).

\section{DisCUSSÃO}

O uso da VNIPP tem-se expandido nos últimos anos. Isso se deve em parte à publicação de estudos bem controlados que documentaram as vantagens da VNIPP sobre a abordagem convencional no tratamento da insuficiência respiratória aguda de diversas etiologias ${ }^{(11)}$, como DPOC $^{(6,7)}$, pneumonia grave adquirida na comunidade $e^{(12)}$, infecção por Pneumocystis carinii ${ }^{(13)}$, edema pulmonar cardiogênico ${ }^{(14)}$, insuficiência respiratória pós-extubação ${ }^{(15)}$ ou como técnica de "desmame" da ventilação mecâni$\mathrm{ca}^{(16)}$. As taxas de sucesso com o uso da VNIPP têm sido 
TABELA 2

Modo de uso da VNIPP e evolução dos pacientes segundo os grupos Sucesso (GS) e Falha (GF)

\begin{tabular}{|c|c|c|c|c|}
\hline & GS, $\mathbf{n}=\mathbf{3 7}$ & $G F, n=23$ & Todos, $n=60$ & Valor de $\mathrm{p}^{*}$ \\
\hline \multicolumn{5}{|l|}{ Tipo de máscara } \\
\hline Nasal, n (\%) & $26(70 \%)$ & $13(57 \%)$ & $39(65 \%)$ & 0,28 \\
\hline Facial, n (\%) & $11(30 \%)$ & $10(43 \%)$ & $21(35 \%)$ & \\
\hline \multicolumn{5}{|l|}{ Tipo de ventilador } \\
\hline Gerador de fluxo^, n (\%) & \multirow{2}{*}{$\begin{array}{r}29(78 \%) \\
8(22 \%)\end{array}$} & $13(57 \%)$ & \multirow{2}{*}{$\begin{array}{l}42(70 \%) \\
18(30 \%)\end{array}$} & 0,072 \\
\hline Convencional ${ }^{\dagger}, \mathrm{n}(\%)$ & & $10(43 \%)$ & & \\
\hline IPAPmax, $\mathrm{cmH}_{2} \mathrm{O}$ & $13,2 \quad 3$ & 114 & 12,3 & 0,020 \\
\hline EPAPmax, $\mathrm{cmH}_{2} \mathrm{O}$ & $5,8 \quad 2$ & $5,4 \quad 1$ & 5,6 & 0,45 \\
\hline Duração da VNIPP (dias) & $3,4 \quad 2,5$ & $2,3 \quad 2$ & 32,3 & 0,098 \\
\hline$<24 \mathrm{~h} \mathrm{n}(\%)$ & $7(19 \%)$ & $13(57 \%)$ & $17(28 \%)$ & 0,003 \\
\hline de 24 a $72 h, n(\%)$ & $17(24 \%)$ & $4(17 \%)$ & $15(25 \%)$ & 0,024 \\
\hline$>72 \mathrm{~h} \mathrm{n}(\%)$ & $13(57 \%)$ & $6(26 \%)$ & $28(47 \%)$ & 0,464 \\
\hline \multicolumn{5}{|l|}{ Complicações } \\
\hline Lesão de pele, n (\%) & \multirow{6}{*}{$\begin{array}{c}4(11 \%) \\
0\end{array}$} & $1 \quad(4 \%)$ & \multirow{6}{*}{$\begin{array}{r}5(8 \%) \\
23(38 \%)\end{array}$} & NS \\
\hline Intubação e causas & & $23(100 \%)$ & & \\
\hline Hipersecreção, n (\%) & & $2 \quad(9 \%)$ & & \\
\hline Torpor, n (\%) & & $3(13 \%)$ & & \\
\hline Agitação psicomotora, n (\%) & & $4 \quad(17 \%)$ & & \\
\hline Piora da doença de base, $\mathrm{n}(\%)$ & & $14(61 \%)$ & & \\
\hline Óbitos, n (\%) & 0 & $10 \quad(43 \%)$ & $10(17 \%)$ & $<0,01$ \\
\hline
\end{tabular}

IPAPmax - máximo valor de pressão inspiratória positiva na via aérea; EPAPmax - máximo valor de pressão expiratória positiva na via aérea; *GS versus GF; $\triangle$ Bilevel $^{\circledast}$; ${ }^{\dagger}$ Bird 8400 , Bird 6400 e Inter 5.

bastante variáveis. Em extensa revisão da literatura, Meduri relacionou 776 pacientes adultos em insuficiência respiratória aguda em 27 trabalhos que avaliaram o uso da VNIPP(3). Desse total, 560 pacientes obtiveram sucesso (72\%) variando de 40 a $93 \%$ dentre os vários estudos. No Brasil, Pinheiro et al.(9), avaliando a VNIPP em protocolo semelhante ao empregado no presente trabalho, obtiveram sucesso em 15 (60\%) de 25 pacientes em insuficiência respiratória de diversas etiologias. $\mathrm{O}$ presente trabalho confirma dados da literatura e recente estudo retrospectivo realizado em nosso serviço, em que a VNIPP constituiu técnica eficiente em tratar a insuficiência respiratória aguda decorrente de diversas etiologias, evitando a intubação em torno de $60 \%$ das vezes ${ }^{(10)}$. Poucos trabalhos encontraram resultados negativos com o uso da VNIPP. Wood et al. ${ }^{(17)}$ compararam o uso da VNIPP à abordagem convencional para o tratamento da insuficiência respiratória aguda em 27 pacientes admitidos em pronto-socorro. Houve maior mortalidade no grupo da VNIPP (25\%) em comparação com o grupo controle $(0 \%)$, sem diferenças quanto à necessidade de intubação (ao redor de $44 \%$ nos dois grupos). A intubação no grupo da VNIPP foi mais tardia do que no grupo controle, o que poderia ter com- prometido a evolução desses pacientes. Dados referentes ao modo de uso da VNIPP, como níveis de IPAP e EPAP utilizados, não foram apresentados. Os autores recomendaram que a VNIPP deveria ser conduzida de forma bastante criteriosa e com seleção adequada de seus candidatos.

Com relação aos dados dos pacientes antes da instituição da VNIPP, verificamos que a falha se associou mais à gravidade da doença demonstrada por escore de Apache Il elevado do que ao diagnóstico de base. Outros autores já demonstraram que pacientes mais graves têm menor probabilidade de sucesso e que o escore de Apache II elevado se associa à falha da VNIPP(18-20), embora outros trabalhos não tenham confirmado essa associação ${ }^{(21,22)}$.

No grupo Sucesso a redução da freqüência respiratória foi mais intensa do que no grupo Falha. Achamos que esse simples parâmetro, quando conjugado com outros sinais clínicos de conforto respiratório (menor uso de musculatura acessória, alívio da dispnéia), melhora do nível de consciência com despertar nos casos de narcose por hipercapnia ou diminuição da agitação psicomotora nos pacientes sem hipercapnia, pode indicar boa resposta à VNIPP. Ao contrário das alterações gasométricas que 
podem levar horas até serem detectadas mudanças significativas ${ }^{(3)}$, esses sinais podem ser avaliados poucos minutos após o início da VNIPP.

A melhora da $\mathrm{PaO}_{2}$ ocorreu em praticamente todos os pacientes, não se associando com a eficiência da VNIPP em prevenir a intubação e/ou a reintubação traqueal. É possível que em muitos casos a correção da hipoxemia não se tenha acompanhado de melhora concomitante da mecânica pulmonar, terminando por não reduzir significativamente o trabalho respiratório. Nesses casos uma possível ineficiência da VNIPP em reduzir o trabalho respiratório poderia explicar a falta de associação entre melhora da $\mathrm{PaO}_{2}$ e a prevenção da intubação. Esse resultado confirma os achados por Wysocki et al. ${ }^{(23)}$ ao verificarem que a variação da $\mathrm{PaO}_{2}$ após 1 h de VNIPP não tivera valor preditivo quanto ao sucesso da VNIPP. Não podemos descartar, contudo, que a $\mathrm{PaO}_{2}$ ou mesmo a relação $\mathrm{PaO}_{2} / \mathrm{FIO}_{2}$ possa ser considerada como um bom parâmetro de resposta naqueles pacientes em que a VNIPP apresenta desempenho inicial satisfatório e a intubação se faz necessária somente após 48-72h. Smyth et al.(20), entretanto, verificaram que em pacientes com DPOC os níveis de $\mathrm{PaO}_{2}$ diferiram entre o grupo Sucesso e Falha somente após $4 \mathrm{~h}$ de VNIPP. Eles determinaram que impossibilidade de manter a $\mathrm{PaO}_{2}$ acima de $60 \mathrm{mmHg}$ após $4 \mathrm{~h}$ de VNIPP se associou a taxa de falha de $68 \%$. Na maioria das vezes não nos foi possível determinar a relação $\mathrm{PaO}_{2} / \mathrm{FIO}_{2}$ durante o uso da VNIPP, uma vez que empregamos com maior freqüência um ventilador gerador de fluxo. Nessa situação o oxigênio foi administrado por fluxo externo, impossibilitando a determinação precisa da $\mathrm{FIO}_{2}$.

Observamos que, nos pacientes em insuficiência respiratória com hipercapnia e acidose, redução da $\mathrm{PaCO}_{2}$ e aumento do $\mathrm{pH}$ após $2 \mathrm{~h}$, mesmo não os normalizando na maioria dos casos, se associaram ao sucesso da VNIPP. Nos pacientes do grupo Falha ocorreu estabilização ou mesmo aumento da $\mathrm{PaCO}_{2}$ com agravamento da acidose, ao mesmo tempo que menor redução da freqüência respiratória. Esses resultados confirmam achados de outros autores. Meduri et al. ${ }^{(22)}$ determinaram que pacientes com hipercapnia que apresentaram redução da $\mathrm{PaCO}_{2}$ $e$ aumento do $\mathrm{pH}$ após 1 a $2 \mathrm{~h}$ em VNIPP obtiveram melhor resposta com menor tempo de assistência ventilatória. Soo Hoo et al. ${ }^{(18)}$ analisaram 14 episódios de insuficiência respiratória hipercápnica em 12 pacientes com DPOC tratados com VNIPP, com sucesso de $50 \%$. A capacidade de adaptar-se à máscara nasal utilizada no estudo e correção mais rápida da freqüência respiratória, da $\mathrm{PaCO}_{2}$ e do $\mathrm{pH}$ se associaram ao sucesso na VNIPP. Ambrosino et al. ${ }^{(19)}$ verificaram que em 59 episódios de uso da VNIPP em 47 pacientes com DPOC a persistência de acidose respiratória mais grave durante a fase inicial da VNIPP se associou ao insucesso. Anton et al. ${ }^{(24)}$, em re- cente estudo prospectivo, determinaram que baixos valores de $\mathrm{VEF}_{1}$ e nível de consciência comprometido à admissão se associaram à falha da VNIPP em 44 episódios de insuficiência respiratória em 36 pacientes DPOC. Além disso, melhora significativa no $\mathrm{pH}$ e na $\mathrm{PaCO}_{2}$ após $1 \mathrm{~h}$ em VNIPP se associou ao sucesso. Os autores, através de um modelo de regressão logística, desenvolveram uma equação para determinação de falha ou sucesso da VNIPP. Eles validaram posteriormente o modelo em outros 15 episódios de insuficiência respiratória aguda com hipercapnia, com acerto em predizer o sucesso da VNIPP em 14 dos 15 episódios. Diaz et al. ${ }^{(25)}$ demonstraram que em pacientes com hipercapnia a VNIPP leva à redução da $\mathrm{PaCO}_{2}$ por aumento da ventilação alveolar, com redução da freqüência respiratória e aumento do volume corrente. Nos pacientes do GF com hipercapnia a VNIPP foi menos eficiente em aumentar ventilação alveolar do que no GS. Consideramos que um dos principais fatores responsáveis por esse fato foram as dificuldades de adaptação dos pacientes desse grupo às interfaces e aos ventiladores utilizados, em especial aos microprocessados convencionais. Além de pacientes mais graves, muitos eram idosos e necessitaram remover suas próteses dentárias, aumentando a probabilidade de vazamento pelos cantos da boca, mesmo em uso de máscara facial, comprometendo a "ciclagem" da PS nos ventiladores convencionais. Tal fato acarretou dessincronia paciente-ventilador e conseqüente agitação psicomotora. Nessa situação, qualquer aumento da PS apenas aumentava o vazamento, agravando o problema. Achamos que isso pode explicar em parte os menores níveis de IPAP alcançados no GF. Meduri et al. ${ }^{(22)}$, em um estudo que incluiu 74 pacientes em insuficiência respiratória com hipercapnia, atribuíram a ineficiência da VNIPP em reduzir a $\mathrm{PaCO}_{2}$ e aumentar o $\mathrm{pH}$ a oferta baixa e inadequada de níveis de pressão inspiratória ( $12 \pm 2$ versus $\left.15 \pm 4 \mathrm{cmH}_{2} \mathrm{O}, \mathrm{p}=0,016\right)$. Vale ressaltar que o ventilador gerador de fluxo utilizado no presente estudo não dispunha de monitorização do volume corrente. Sem dúvida, a determinação desse parâmetro poderia ter orientado melhor os ajustes de IPAP titulando os níveis ideais. Achamos que novos trabalhos abordando VNIPP procurem responder até quanto e quando devemos persistir tentando otimizar a VNIPP nos casos em que há má resposta inicial.

Com relação à forma de utilizar a VNIPP houve maior uso de máscaras nasais e de ventiladores geradores de fluxo no GS em relação ao GF, mas sem significância estatística (Tabela 2). Consideramos, portanto, improvável que o tipo de máscara ou de ventilador usado tenham sido os principais responsáveis pelos casos de falha da VNIPP $e$ que a maior gravidade dos pacientes do GF tenha sido o fator mais relevante. Preferimos a utilização inicial de máscaras nasais por proporcionarem maior conforto, me- 
nor sensação de claustrofobia e maior aceitação na fase inicial da VNIPP. Sua grande desvantagem ficou evidente nos pacientes com respiração bucal ou naqueles que não conseguiam manter a boca fechada à medida que eram elevadas as pressões inspiratórias e expiratórias. Nesses casos a mudança imediata para a máscara facial se fez necessária. O uso de ventilador gerador de fluxo proporcionou maior sincronia paciente-ventilador, uma vez que pequenos vazamentos não comprometiam a "ciclagem" do aparelho. Sua principal limitação é a impossibilidade de ofertar $\mathrm{FIO}_{2}>50 \%$ e a falta de monitorização do volume corrente. Nos casos de hipoxemia grave foi necessário mudar para ventiladores convencionais. Como exemplo, citamos uma de nossas pacientes com pneumonia por Pneumocystis carinii que necessitou de $\mathrm{FIO}_{2}$ de $100 \%$ e máscara facial pelo menos por cinco dias, tendo permanecido 14 dias no total em VNIPP.

As intubações no GF ocorreram em sua maioria nas primeiras 24 h após o início da VNIPP. Consideramos que a identificação precoce de falha é importante para o manejo adequado da insuficiência respiratória, evitando-se protelar a intubação traqueal, e que esse fator possivelmente tem impacto significativo na evolução dos pacientes $^{(17)}$. Cerca de $1 / 4$ dos pacientes foi intubada somente após três dias de uso da VNIPP, configurando "falha tardia". As razões para tanto podem ter sido: incapacidade em reconhecer precocemente a falha da VNIPP ou resposta inicial positiva à VNIPP, porém com agravamento posterior da insuficiência respiratória. Moretti et al. ${ }^{(26)}$, em recente estudo multicêntrico na Itália, avaliaram 137 pacientes que inicialmente responderam à VNIPP. Desse total, $31(23 \%)$ apresentaram novo episódio de insuficiência respiratória aguda enquanto ainda estavam em VNIPP $(8,4 \pm 2,8$ dias). A mortalidade dos pacientes que seguiram na VNIPP em vez da intubação traqueal foi de $92 \%$ e superior aos $53 \%$ de óbitos do grupo que foi intubado. Os autores concluíram que pacientes DPOC têm probabilidade de $20 \%$ de apresentar um segundo episódio de insuficiência respiratória aguda após boa resposta inicial à VNIPP e que deve ser considerada sua interrupção nesses casos de falha tardia. Mais estudos prospectivos analisando as falhas tardias da VNIPP são necessários.

Verificamos alta mortalidade no grupo em que houve falha da VNIPP (10 de 23, 43\%) e nenhum óbito no grupo Sucesso. Esses resultados são semelhantes aos de Pinheiro et al. ${ }^{(9)}$, que observaram seis óbitos em sete pacientes em que a VNIPP falhou e nenhum no grupo que obteve sucesso. Em parte a elevada mortalidade pode ser explicada pela maior gravidade dos pacientes do GF. Devemos considerar, por outro lado, que possíveis complicações decorrentes da intubação traqueal e da ventilação mecânica convencional per se tenham contribuído para essa diferença de mortalidade entre os grupos. Foram poucas as complicações da VNIPP, sendo a mais freqüente o desenvolvimento de úlceras de pele nos locais de contato da máscara com o nariz em $8 \%$ dos pacientes, à semelhança de outros trabalhos da literatura ${ }^{(3)}$.

Portanto, achamos que a VNIPP constituiu uma alternativa segura e eficaz no tratamento da insuficiência respiratória aguda de diversas etiologias, evitando a intubação traqueal em um número significativo de casos $162 \%$ nesta série).

Escore de Apache II elevado, redução apenas discreta da freqüência respiratória e uso de níveis relativamente mais baixos de IPAP associaram-se à falha da VNIPP em prevenir a intubação traqueal. A melhora da $\mathrm{PaO}_{2}$ não se associou ao sucesso da VNIPP. Por outro lado, nos pacientes com hipercapnia e acidose respiratória, o aumento do $\mathrm{pH}$ acompanhado de redução da $\mathrm{PaCO}_{2}$ em intervalo de até $2 \mathrm{~h}$ foi associado ao seu sucesso.

A falha da VNIPP associou-se à elevada mortalidade. A identificação imediata dos casos em que ela ocorre deve ser estimulada para não se protelar a intubação traqueal. Nos casos de sucesso ocorreu número reduzido de complicações.

O presente trabalho reforça o entusiasmo com o uso da VNIPP. Contudo, para que sua taxa de sucesso se eleve sem que haja comprometimento da evolução dos pacientes nos casos de falha, sua implementação deve seguir critérios rigorosos quanto a: indicação, seleção dos pacientes e seu modo de uso. É preciso que se determinem critérios objetivos de resposta para aproveitamento real de suas vantagens em relação à ventilação convencional.

\section{AgradeCimEnTOS}

À equipe de enfermagem da UTI respiratória do Hospital de Messejana.

Ao Dr. José Dumas Ferreira Gomes, Chefe Clínico da UTI respiratória do Hospital de Messejana.

\section{REFERÊNCIAS}

1. II Consenso Brasileiro de Ventilação Mecânica. J Pneumol 2000;26: S60-S63.

2. Meduri GU. Noninvasive positive-pressure ventilation in patients with acute respiratory failure. Clin Chest Med 1996;513-553.

3. Meduri GU. Noninvasive positive-pressure ventilation in patients with acute respiratory failure. In: Marini JJ, Slutsky AS, eds. Physiological basis of ventilatory support. New York: Marcel Dekker, Inc., 1998; 921-996.

4. Barach AL, Martin J, Eckman M. Positive pressure respiration and its application to the treatment of acute pulmonary edema. Ann Intern Med 1938;12:754-795.

5. Brochard L, Isabey D, Piquet J, et al. Reversal of acute exacerbations of chronic obstructive lung disease by inspiratory assistance with a face mask. N Engl J Med 1990;323:1523-1530.

6. Brochard L, Mancebo J, Wysocki M, et al. Noninvasive ventilation for acute exacerbations of chronic obstructive pulmonary disease. N Engl J Med 1995;333:817-822. 
7. Bott J, Carroll MP, Conway JH, et al. Randomized controlled trial of nasal ventilation in acute ventilatory failure due to chronic obstructive airways disease. Lancet 1993;341:1555-1557.

8. Keenan SP, Kernerman PD, Cook DJ, Martin CM, McCormack D, Sibbald WJ. Effect of noninvasive positive pressure ventilation on mortality in patients admitted with acute respiratory failure: a meta-analysis. Crit Care Med 1997;25:1685-1692.

9. Pinheiro BV, Pinheiro AF, Henrique DMN, Oliveira JCA, Baldi J. Ventilação não-invasiva com pressão positiva em pacientes com insuficiência respiratória aguda. J Pneumol 1998;24:23-29.

10. Holanda MA, Rocha EM, Bandeira RM, Aguiar IV, Leal W, Oliveira $\mathrm{CH}$. Uso e eficiência da ventilação não-invasiva em pacientes com insuficiência respiratória de diversas etiologias. J Pneumol 1998;24: S63.

11. Martin TJ, Jeffrey DH, Constantino JP, et al. A randomized prospective evaluation of noninvasive ventilation for acute respiratory failure. Am J Respir Crit Care Med 2000;161:807-813.

12. Confalonieri M, Potena A, Carbone G, Porta RD, Tolley EA, Meduri GU. Acute respiratory failure in patients with severe community-acquired pneumonia. A prospective randomized evaluation of noninvasive ventilation. Am J Respir Crit Care Med 1999;160:1585-1591.

13. Miller RF, Semple SJG. Continuous positive airway pressure ventilation for respiratory failure associated with Pneumocystis carinii pneumonia. Respir Med 1991;85:133-138.

14. Bersten AD, Holt AW, Vedig AE, Skowronski GA, Baggoley CJ. Treatment of severe cardiogenic pulmonary edema with continuous positive airway pressure delivered by a face mask. N Engl J Med 1991;325: 1825-1830.

15. Rosinha SRPO, Lobo SMA, Sanches HS, et al. Noninvasive positive pressure ventilation can prevent reintubation after acute respiratory failure: results of a prospective randomized study. Am J Respir Crit Care Med 2000;161:A262.

16. Nava S, Ambrosino N, Clini E, et al. Noninvasive mechanical ventilation in the weaning of patients with respiratory failure due to chronic obstructive pulmonary disease. A randomized, controlled trial. Ann Intern Med 1998;128:721-728.
17. Wood KA, Lewis L, Von Harz B, Kollef, MH. The use of noninvasive positive pressure ventilation in the emergency department. Results of a randomized clinical trial. Chest 1998;113:1339-1346.

18. Soo Hoo GW, Santiago S, Williams AJ. Nasal mechanical ventilation for hypercapnic respiratory failure in chronic obstructive pulmonary disease: determinants of success and failure. Crit Care Med 1994;22: 1253-1261.

19. Ambrosino N, Foglio K, Rubini F, Clini E, Nava S, Vitacca M. Noninvasive mechanical ventilation in acute respiratory failure due to chronic obstructive pulmonary disease: correlates for success. Thorax 1995; 50:755-757.

20. Smyth CM, Wilkinson M, White V, Angus RM. Early indicators of outcome of non-invasive ventilation in exacerbations of chronic obstructive pulmonary disease. Am J Respir Crit Care Med 2000;161:A262.

21. Fernandez R, Blanch LI, Valles J, Baigorri F, Artigas A. Pressure support ventilation via face mask in acute respiratory failure in hypercapnic COPD patients. Intensive Care Med 1993;19:456-461.

22. Meduri GU, Turner RE, Abou-Shala N, Wunderink R, Tolley E. Noninvasive positive pressure ventilation via face mask. First line intervention in patients with acute hypercapnic and hypoxemic respiratory failure. Chest 1996;109:179-193.

23. Wysocki M, Tric L, Wolff MA, Gertner J, Millet H, Herman B. Noninvasive pressure support ventilation in patients with acute respiratory failure. Chest 1993;103:907-913.

24. Anton A, Guell R, Gomez J, et al. Predicting the result of noninvasive ventilation in severe acute exacerbations of patients with chronic airflow limitation. Chest 2000;117:625-627.

25. Diaz O, Iglesia R, Ferrer M, et al. Effects of noninvasive ventilation on pulmonary gas exchange and hemodynamics during exacerbations of chronic obstructive pulmonary disease. Am J Respir Crit Care Med 1997; 156:1840-1845.

26. Moretti M, Cilione C, Tampieri A, Fracchia C, Marchioni A, Nava S. Incidence and causes of non-invasive mechanical ventilation failure after initial success. Thorax 2000;55:819-825. 